\title{
X-Ray Structural Studies of $(\mathbf{1} \rightarrow \mathbf{3})-\boldsymbol{\beta}$-D-Glucan (Curdlan)
}

\author{
Hirofumi TAKedA, ${ }^{* 1}$ Naritake YASUOKA, ${ }^{* 1}$ Nobutami KASAI, ${ }^{*, * 1}$ \\ and Tokuya HARADA ${ }^{* 2}$ \\ *1Department of Applied Chemistry, Faculty of Engineering, \\ Osaka University, Yamadakami, Suita, Osaka 565, Japan. \\ ${ }^{*}$ Institute of Scientific and Industrial Research, Osaka University, \\ Yamadakami, Suita, Osaka 565, Japan.
}

(Received November 7, 1977)

\begin{abstract}
KEY WORDS (1 $\rightarrow 3)-\beta$-D-Glucan / X-Ray Diffraction / Curdlan / Single Helical Structure / Triple Helical Structure / Micelle / Stretched / Water-Swollen / Annealed /
\end{abstract}

The structural feature and biological significance of $(1 \rightarrow 3)-\beta$-D-glucan (curdlan) are subjects of increasing interest. ${ }^{1}$ It has been reported that curdlan $13140^{2}$ is essentially a linear molecule composed exclusively of $(1 \rightarrow 3)$ - $\beta$-linked D-glucose residues. ${ }^{3}$ Recent $\mathrm{X}$-ray work on $(1 \rightarrow 3)$ - $\beta$-D-glucan has given many interesting results, ${ }^{4-6}$ and the triple-stranded helical structure of the molecule has been proposed for the annealed fiber. ${ }^{6}$ The triple helix was already proposed for the $(1 \rightarrow 3)-\beta$-D-xylan molecule. ${ }^{7}$ This paper describes the results of X-ray diffraction studies of the $(1 \rightarrow 3)-\beta$-D-glucan, and proposes a single helical structure of the molecule at room temperature.

A film specimen regenerated from an alkaline solution of the curdlan $13140^{2}\left(\overline{\mathrm{DP}_{n}}, 400\right)$ was subjected to water swelling treatment at room temperature. A fibrous specimen was obtained by stretching the swollen film uniaxially (draw ratio, ca. $3.5: 1$ ). The wet, fibrous specimens with dimensions, $c a .0 .3 \times 0.5 \times 10 \mathrm{~mm}^{3}$ were sealed in thin-walled glass capillary tubes, and examined by the wide- and small-angle X-ray diffractions. Flat and cylindrical film cameras and a small-angle scattering apparatus were used with nickel-filtered $\mathrm{Cu}-K \alpha$ radiation.

Figure 1 shows an X-ray fiber diagram of the wet, fibrous specimen. It is noteworthy that the center of the fiber diagram has a cross-like appearance, suggesting a simple helical structure

\footnotetext{
* To whom correspondence should be addressed.
}

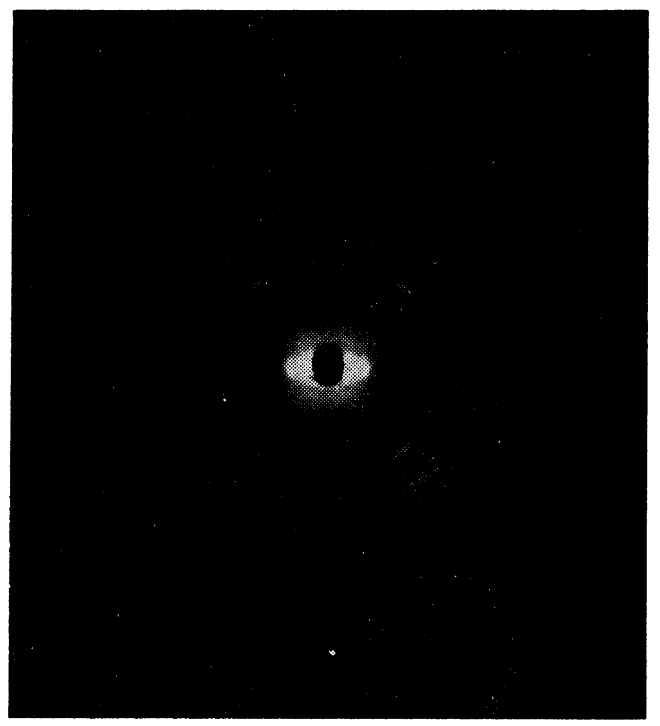

Figure 1. X-Ray diffraction diagram of the wet, fibrous specimen of the $(1 \rightarrow 3)-\beta$-D-glucan (curdlan 13140). (Flat camera, $\mathrm{Cu}-K \alpha$, fiber axis vertical.)

for the molecule. The other feature of the fiber diagram is that the average intensities of the layers 1 and 2 were much weaker than those of the layers 0,3 , and 4 . A total of 17 observed reflections could be indexed by an orthorhombic unit-cell with $a=26.4, b=16.4$, and $c=22.6_{5} \AA$ (fiber axis); however, the corresponing space group could not be determined.

Reasonable models of $7 / 1$ and $6 / 1$ single helices with the fiber period $22.6_{5} \AA$ could be 
constructed using the coordinate of the standard $\beta$-D-glucose residue $^{8}:$ the conformation of the $\beta-(1 \rightarrow 3)$ linkage being $(\Phi, \Psi)=\left(46.5^{\circ}, 6^{\circ}\right)(6 / 1$ right-handed), $\left(-8^{\circ},-50^{\circ}\right)(6 / 1$ left-handed $)$, $\left(39^{\circ},-1^{\circ}\right)(7 / 1$ right-handed $)$ and $\left(-1^{\circ},-42^{\circ}\right)$ (7/1 left-handed) according to the definition of

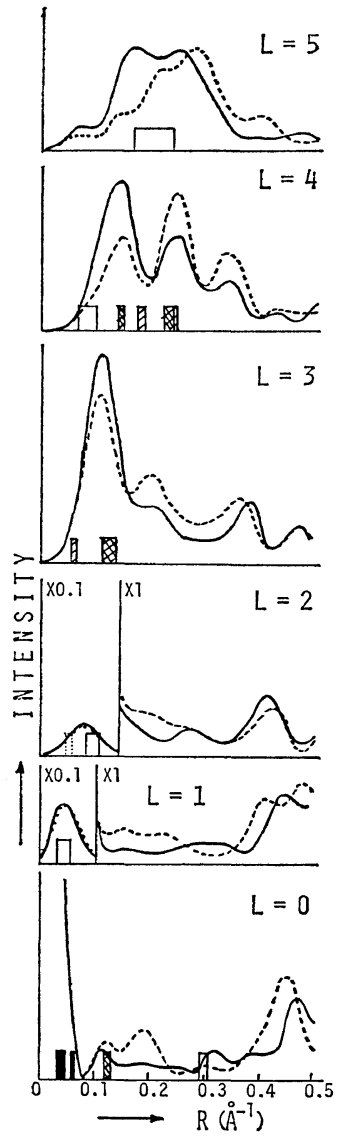

(a)

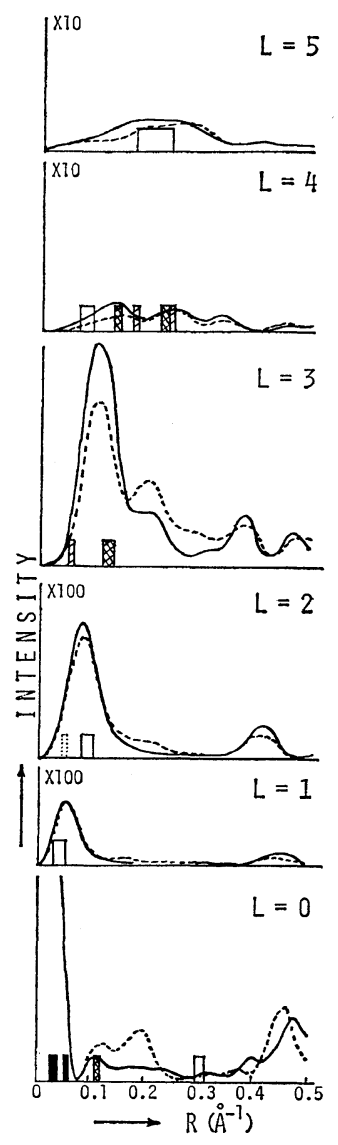

(b)
Figure 2. (a) The Fourier transforms of the righthanded $(-)$ and left-handed $(--)$ 7/1 single helical models of the $(1 \rightarrow 3)-\beta$-D-glucan molecule calculated by the equation of Klug, et al. ${ }^{10}$ On each layer line, the observed feature of the diffraction pattern is marked by a filled, cross-shaded, shaded or unfilled rectangle or a broken-line rectangle according to the relative intensity of the pattern, very strong, strong, medium, weak, or very weak, respectively. (b) The Fourier transforms of the right-handed $(-)$ and left-handed $(--)$ ) $7 / 1$ triple-stranded helices, of which coordinates are $(R, \phi, Z),\left(R, \phi \pm 106.5^{\circ}, Z+0.85 \AA\right)$, and $\left(R, \phi \pm 213^{\circ}, Z+1.7 \AA\right)$.
Sathyanarayana and $\mathrm{RaO}^{9}$ and the linkage angle being fixed as $117.5^{\circ}$. All of these conformations are located in relatively low energy regions in the conformational energy map of the $(1 \rightarrow 3)-\beta$-D-glucan. ${ }^{9}$

The Fourier transform of each helix was calculated using the equation given by Klug, et al. ${ }^{10}$ The atomic scattering factors used for $\mathrm{C}$ and $\mathrm{O}$ atoms were taken from Cromer and Waber ${ }^{11}$ and were later corrected for the effect of the water immersing the molecules as was carried out in the X-ray analysis of DNA. ${ }^{12}$ This result is presented in Figure 2(a) together with the relative magnitude of the observed intensities. As seen in Figure 2(a) the $7 / 1$ single helix, either left- or right-handed, agrees with the observed peak positions of the layers 0,1 , 2 , and 3 better than those of the $6 / 1$ single helix. However, the calculated intensities of layers 1 and 2 are larger than the observed intensities. The density of the specimen was determined by the flotation method in a solution of benzene/carbon tetrachloride and was $1.17 \mathrm{~g} \mathrm{~cm}^{-3}$. This value and the unit-cell dimensions determined the number of glucose residues per unit-cell as 14: two molecular chains and $c a .250$ water molecules being contained per unit-cell.

The Fourier transforms of the double- and triple-stranded $7 / 1$ helices were also calculated by using the equation given by Fraser and MacRae. ${ }^{13}$ Among these, a triple-stranded helix having the coordinates, $(R, \phi, Z),\left(R, \phi \pm 106.5^{\circ}\right.$, $Z+0.85 \AA)$ and $\left(R, \phi \pm 213^{\circ}, Z+1.7 \AA\right)$ showed a better fit with the observed peak positions (Figure 2(b)). However, the calculated intensities of the layers 1 and 2 are very weak, about one-hundredth of that of the equater.

These facts suggest that most of the $(1 \rightarrow 3)$ $\beta$-D-glucan molecules in the wet fibrous specimen have a single $7 / 1$ helical and the rest have a triple-stranded $7 / 1$ helical structures. This result is consitent with the conclusion of a ${ }^{13} \mathrm{C}-\mathrm{NMR}$ study of the $(1 \rightarrow 3)$ - $\beta$-D-glucan gel. ${ }^{14,15}$

The small-angle $\mathrm{X}$-ray scattering photograph of the wet specimen shows only a diffuse streak along the equator (Figure 3). Assuming that the inhomogeneity in the specimen consists of highly dispersed, uniform, long cylindrical micelles which are arranged parallel to the 


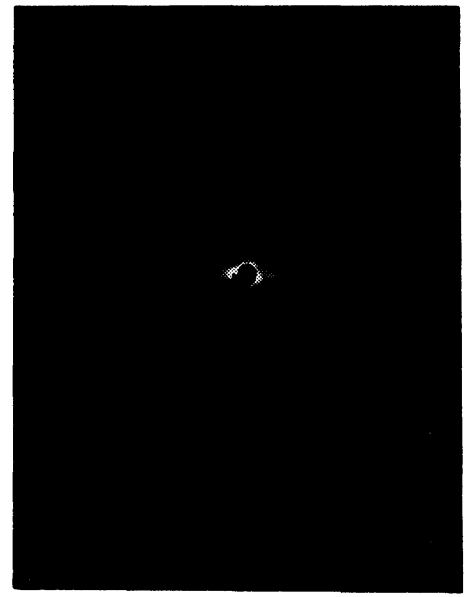

Figure 3. The small-angle X-ray scattering photograph of the wet, fibrous specimen. (Cu-Ko, fiber axis vertical.)

stretching direction, the radius of the micelles was estimated to be $c a$. $40 \AA$ by the Guinier plot of the intensity distribution along the equator. The $\varepsilon^{2} I-\varepsilon$ plot gives a measure of intermicellar distance in the equator as $c a$. $120 \AA$, where $I$ and $\varepsilon$ are the scattering intensity and the scattering angle, respectively.

Figure 4 gives a variation of X-ray fiber diagrams, taken at regular intervals, of a wet, fibrous specimen dried in the air at room temperature. During the course of drying, the broadening of each pattern destroyed the detailes of the fiber diagram (Figure $4(a)-(d)$ ); 013,023 and 224 reflections fused into a broad pattern, from which the fiber period was determined as ca. $6 \AA$, and the small-angle scattering disappeared. These facts suggest that the micelles lose intramicellar water (or crystalline water) by drying, which disturbs the regular molecular arrangement inside the micelle, and at the same time, the evapolation of intermicellar water causes adjacent micelles to contact directly, which does away with the inhomogeneity in the specimen.

The wet fibrous specimen was then subjected to heat-treatment in a sealed bomb at ca. 100 to $130^{\circ} \mathrm{C}$ in the presence of the water. At temperatures above $120^{\circ} \mathrm{C}$, the X-ray fiber diagram of the annealed specimen (Figure 5(a)) showed a remarkable difference from that of the original one: the first layer disappeared, and the fiber period decreased to $18.12 \AA$. After the heattreatment the specimen never showed the waterswelling behavior suggesting the existence of a compact hydrophorbic structure. The annealed specimen dried in vacuum gave another different X-ray diagram, having a fiber period of $5.7_{2} \AA$ (Figure 5(b)). These two kinds of diffraction diagrams are similar to those observed by Marchessault, et al., ${ }^{6}$ with specimens prepared from DMSO solution and annealed at $143^{\circ} \mathrm{C}$, by which the triple-stranded helical structure of the molecule was evaluated..$^{6,16}$ Considering this with the results at room temperature, it can be concluded that by heattreatment, the single helical molecules change their structures to the triple-stranded helices.

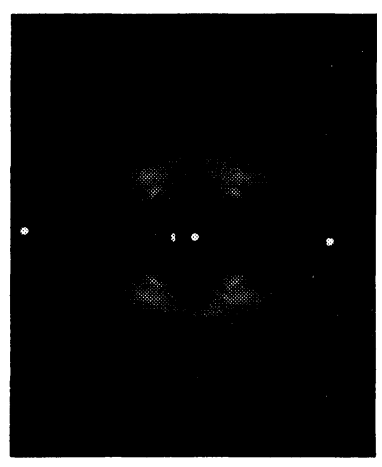

(a)

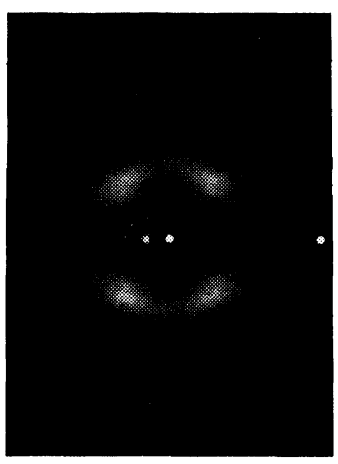

(b)

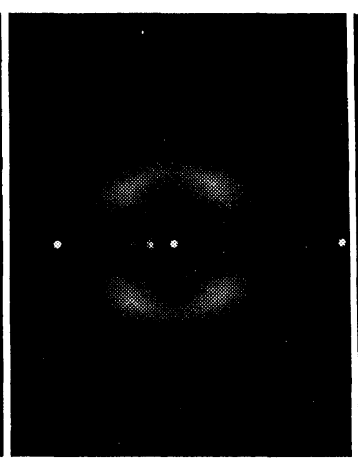

(c)

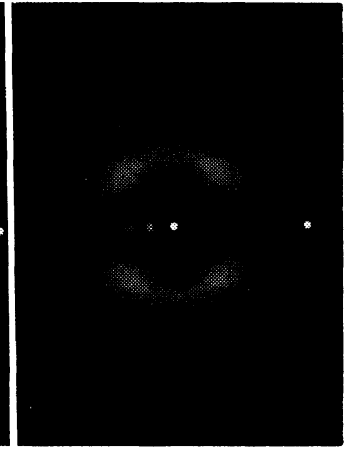

(d)

Figure 4. The variation of the X-ray fiber diagrams of the wet, fibrous specimen dried in the air. The photographs were taken at regular intervals $(1 \mathrm{hr})$. (Cylindrical camera, Co-K $\alpha$, fiber axis vertical.) 


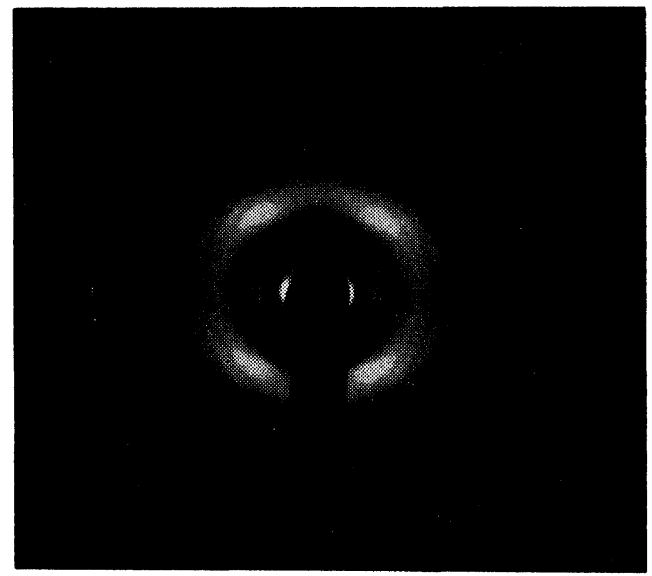

(a)

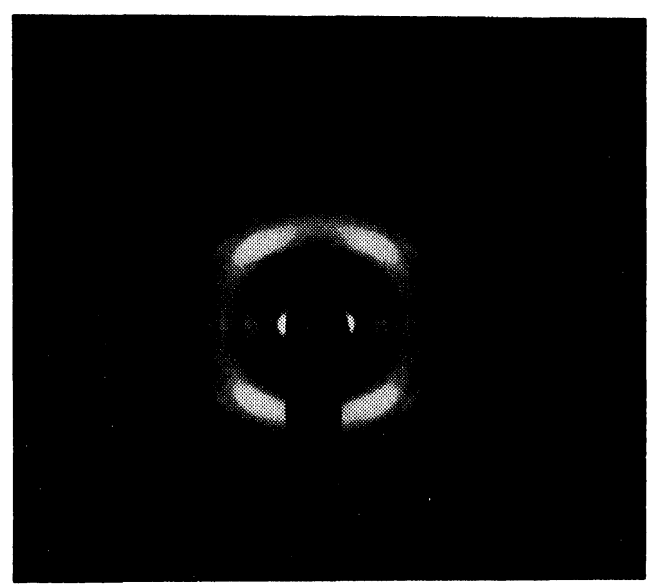

(b)

Figure 5. (a) The X-ray fiber diagram of the specimen dried in air after heat treatment above $120^{\circ} \mathrm{C}$. (Cylindrical camera, Cu-K $\alpha$, fiber axis vertical.) (b) The X-ray fiber diagram of the annealed specimen dried in vacuum at $60^{\circ} \mathrm{C}, 3 \mathrm{hr}$. (Cylindrical camera, $\mathrm{Cu}-\mathrm{K} \alpha$, fiber axis vertical.)

Acknowledgment. The authors should like to express their appreciation to Takeda Chemical Industries, Ltd. for providing the Curdlan 13140 .

\section{REFERENCES}

1. T. Harada, A. Misaki, and H. Saito, Arch. Biochem. Biophys., 124, 292 (1963); T. Harada, "Ferment. Technology Today," G. Terui Ed., Society of Fermentation Technology, Japan, 1972, p 603.

2. T. Harada, Process. Biochemistry, 9, 21 (1974).

3. H. Saito, A. Misaki, and T. Harada, Agric. Biol. Chem., 32, 1261 (1968).

4. J. Jelsma and D. R. Kreger, Carbohydr. Res., 43, 200 (1975).

5. T. L. Bluhm and A. Sarko, Biopolymers, 14, 2639 (1975).

6. R. H. Marchessault, Y. Deslandes, K. Ogawa, and P. R. Sundarajan, Can. J. Chem., 55, 300
(1977).

7. E. D. T. Atkins and K. D. Parker, J. Polym. Sci., Part C, 28, 69 (1969).

8. S. Arnott and W. E. Scott, J. Chem. Soc. Perkin Trans. 2, 324 (1972).

9. B. K. Sathyanarayana and V. S. R. Rao, Biopolymers, 10, 1605 (1971).

10. A. Klug, F. H. C. Crick, and H. W. Wyckoff, Acta Crystallogr., 11, 199 (1958).

11. D. T. Cromer and J. T. Waber, Acta Crystallogr., 18, 104 (1965).

12. S. Arnott and D. W. L. Hukins, J. Mol. Biol., 81, 93 (1973).

13. R. D. B. Fraser, T. P. MacRae, and A. Miller, Acta Crystallogr., 17, 769 (1964).

14. H. Saito, T. Ohki, Y. Yoshioka, and F. Fukuoka, FEBS Lett., 68, 15 (1976).

15. H. Saito, T. Ohki, and T. Sasaki, Biochemistry, 16, 908 (1977).

16. T. L. Bluhm and A. Sarko, Can. J. Chem., 55, 293 (1977). 